\title{
Safety and durability of single-stage type I hybrid total aortic arch repair for extensive aortic arch disease: early- and long-term clinical outcomes from a single center and our 10-year of experience
}

\author{
Bowen Zhang ${ }^{1}$, Yizhen Wei ${ }^{1}$, Yanxiang Liu ${ }^{1}$, Hao Lin $^{2}$, Shenghua Liang ${ }^{1}$, Yaojun Dun ${ }^{1}$, Cuntao Yu ${ }^{1}$, \\ Xiangyang Qian ${ }^{1}$, Hongwei Guo ${ }^{1}$, Xiaogang Sun ${ }^{1}$ \\ ${ }^{1}$ Department of Cardiovascular Surgery, Fuwai Hospital, National Center for Cardiovascular Diseases, Chinese Academy of Medical Sciences and \\ Peking Union Medical College, Beijing, China; ${ }^{2}$ Department of Cardiovascular Surgery, Shandong Provincial Hospital affiliated to Shandong First \\ Medical University, Jinan, China \\ Contributions: (I) Conception and design: B Zhang, Y Wei, Y Liu, Y Dun; (II) Administrative support: X Sun; (III) Provision of study materials or \\ patients: Y Wei, C Yu, X Qian, H Guo, X Sun; (IV) Collection and assembly of data: B Zhang, Y Liu, S Liang; (V) Data analysis and interpretation: \\ H Lin, S Liang, Y Dun; (VI) Manuscript writing: All authors; (VII) Final approval of manuscript: All authors. \\ Correspondence to: Xiaogang Sun. Department of Cardiovascular Surgery, Fuwai Hospital, National Center for Cardiovascular Diseases, Chinese \\ Academy of Medical Sciences and Peking Union Medical College, No. 167 North Lishi Road, Xicheng District, Beijing 100037, China. \\ Email: xiaogangsun1@163.com; xiaogangsun2006@vip.sina.com.
}

Background: Single-stage type I hybrid total aortic arch repair is a surgical treatment for extensive aortic arch disease, but the clinical outcomes were distinguishing. The purposes of this study were to share our experience and evaluate the perioperative safety and long-term durability.

Methods: Thirty-six patients who underwent single-stage type I hybrid total aortic arch repair in Fuwai Hospital between January 2010 and June 2020 were respectively reviewed. Early primary endpoint was defined as early composite adverse events, including mortality, multiple organ dysfunction syndrome (MODS), unplanned reoperation, stroke, paraplegia, acute renal failure (ARF) necessitating continuous renal replacement therapy (CRRT), respiratory failure and stents related complications. Long-term endpoints included late mortality, late aortic related reintervention and late adverse aortic events. When evaluating the early- and long-term outcomes, all patients were stratified into two subgroups by age (65 years).

Results: All patients acquired technical success. Early composite adverse events rate was 11.1\% (4/36), inhospital mortality was $8.3 \%$ (3/36). Average follow-up period was $48.0 \pm 35.3$ months. Overall survival rate was $83.3 \%$ and $51.9 \%$ at 5 and 10 years respectively. Late aortic related reintervention occurred at one $(3.0 \%$, $1 / 33$ ) patient and this patient died after reintervention. Overall freedom from adverse aortic events was $79.2 \%$ and $47.5 \%$ at 5 and 10 years respectively. Significant difference was not observed between the elderly and young subgroups, no matter in early- and long-term outcomes.

Conclusions: Single-stage type I hybrid total aortic arch repair has achieved desirable outcomes in our center, which does not increase perioperative risk in the elderly patients, meanwhile, also acquire acceptable durability in the young patients. In conclusion, this surgery is a practical mini-invasive treatment for extensive aortic arch disease with strict and limited indications.

Keywords: Hybrid technique; total aortic arch repair; extensive aortic arch disease

Submitted Dec 09, 2020. Accepted for publication Feb 24, 2021.

doi: $10.21037 /$ jtd-20-3479

View this article at: http://dx.doi.org/10.21037/jtd-20-3479 


\section{Introduction}

Conventional total aortic arch replacement with vascular prosthesis under hypothermic circulatory arrest (HCA) for extensive aortic arch disease is associated with substantial morbidity and mortality, despite significant advances in surgical technique and perioperative care $(1,2)$. Endovascular technology is a mini-invasive therapeutic method and has been widely used in the lesion of descending aorta (3), but it is hindered to be applied into aortic arch disease due to the presence of supra-arch branches. Recently, hybrid technique that combine surgical supra-arch debranching with concomitant endovascular exclusion of the diseased aortic arch has emerged as a practical treatment for extensive aortic arch disease (4).

There are three types of hybrid total aortic arch repair, called type I, II and III respectively (5). Due to the ascending aorta or the aortic arch replacement in the type II or III hybrid repair, cardiopulmonary bypass (CPB) and even HCA is inevitable, which may cause postoperative complications like conventional surgery, although providing the stable proximal landing zone. Type I hybrid total aortic arch repair is less invasive because $\mathrm{CPB}$ or HCA is theoretically avoided, but the clinical outcomes of this surgery reported by different centers were distinguishing (6-9).

The purposes of this study were to share our 10-year of operative experience and provide the full evaluation of early- and long-term clinical outcomes of single-stage type I hybrid total aortic arch repair for extensive aortic arch disease.

We present the following article in accordance with the TREND reporting checklist (available at http://dx.doi. org/10.21037/jtd-20-3479).

\section{Methods}

\section{Patients}

From January 2010 to June 2020, 637 patients have undergone single-stage hybrid aortic arch repair in the vascular surgery center of Fuwai Hospital. Of these, 36 patients who underwent single-stage type I hybrid total aortic arch repair were included into this cohort. Type I hybrid total aortic arch repair was defined as surgical total supra-arch debranching and zone 0 stent graft deployment to exclude the entire diseased aortic arch.

Four aspects of indications for this surgery were considered. Firstly, extensive arch disease involving the entire aortic arch or involving partial aortic arch but without adequate landing zone between supra-arch branches. Secondly, morphology of ascending aorta is normal, excluding dissection, expansion (diameter $>38 \mathrm{~mm}$ ), and severe aortic calcification, etc. Thirdly, patients were considered to be high risk for $\mathrm{CPB}$ or HCA, such as advanced age or severe comorbidities. Finally, operation costs and surgeons' experience were also important factors to impact the surgical protocol. Hereditary connective tissue disorders, severe malperfusion syndrome, unstable vital signs were listed as contraindications.

\section{Surgical technique}

Surgery was performed in the hybrid operating room equipped with a universal floor mounted angiographic C-arm system (Siemens Medical, Forchheim, Germany) for fluoroscopic guidance during stent graft deployment. This surgery was divided into two portions, surgical supra-arch debranching and endovascular aortic arch repair.

After median sternotomy or upper mini-sternotomy incision, the ascending aorta and the supra-aortic vessels were mobilized. Systemic heparinization is initiated and systolic blood pressure was lowered to $100 \mathrm{mmHg}$. Three patterns of vascular prothesis were used to perform debranching procedure, which was presented in the Figure 1. The ascending aorta is tangentially clamped with a sidebiting clamp and the proximal part of the prosthesis is sutured end-to-side to the ascending aorta. The side-biting clamp is removed and the limbs of graft are anastomosed end-to-end to the supra-arch branches one by one. In the early stages of developing this surgery, left subclavian artery (LSCA) was not reconstructed in five patients for shortening operative time and improving operative safety. $\mathrm{CPB}$ was always prepared for all patients.

After supra-arch debranching and concomitant heart operation, angiography was performed to measure the length and diameter of proximal landing zone and the range of lesion. Retrograde access through femoral artery was routinely used to deploy the stents. If failed, a $10-\mathrm{mm}$ graft was sutured end-to-side to ascending aorta to deploy the stents by antegrade access. Balloon dilatation or another stent deployment will be performed when the endoleaks was noticed. Last angiography was done to check the technical success including the patency of the bypass and the condition of the stents. Six different commercially available devices were implanted: Zenith (Cook Medical Inc., 
A

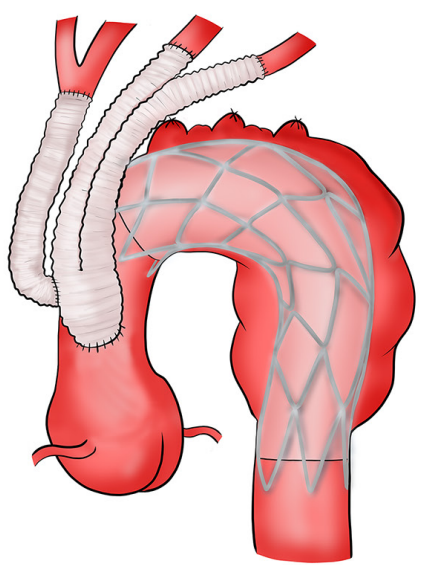

B

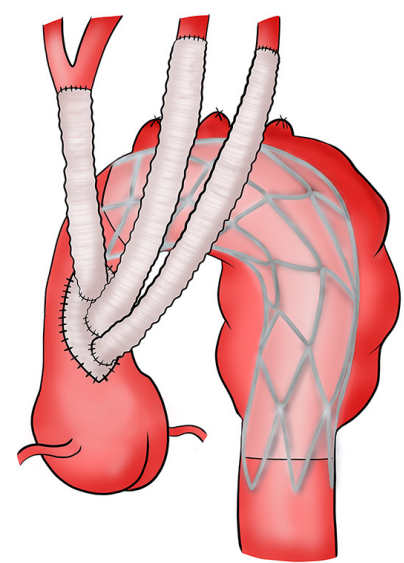

C

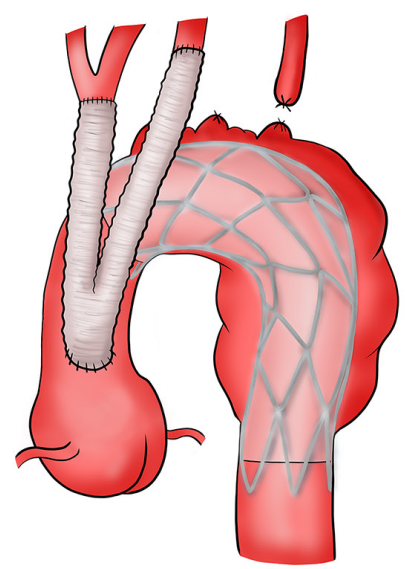

Figure 1 Operative technique of single-stage type I hybrid total aortic arch repair. (A) Patten 1: supra-arch debranching (straight vascular prothesis tailored from branches of "Y" shape vascular prothesis was handsewn end-to-side to the trunk of the "Y" shape Dacron vascular prosthesis (Hemashield Platinum, Maquet Cardiovascular LLC, NJ, USA; $16 \times 8 \times 8 \mathrm{~mm}$ in diameter)) and endovascular aortic repair. (B) Patten 2: supra-arch debranching (4-branched vascular prosthesis (Hemashield Platinum, Maquet Cardiovascular LLC, NJ, USA; $28 \times 10 \times 8 \times 8 \times 10 \mathrm{~mm}$ in diameter) was tailored to an island) and endovascular aortic repair. (C) Patten 3: supra-arch debranching ("Y" shape vascular prosthesis was used to reconstruct innominate artery and left common carotid artery) and endovascular aortic repair.

Bloomington, IN, USA), Relay (Bolton Medical, Sunrise, FL, USA), Valiant Captivia and Endurant (Medtronic Inc., Galway, Ireland), Gore stent graft (W.L. Gore \& Assoc, Flagstaff, AZ, USA) and Hercules (MicroPort Endovascular MedTech Co., Ltd., Shanghai, China).

\section{Data collection and follow-up study}

Demographic data, operative information and postoperative complications were routinely collected. Early primary endpoint was defined as early composite adverse events, which included mortality, multiple organ dysfunction syndrome (MODS), unplanned reoperation, stroke, paraplegia, acute renal failure (ARF) necessitating continuous renal replacement therapy (CRRT), respiratory failure and stents related complications. Postoperative $24 \mathrm{~h}$ drainage, delayed awaking, mechanical ventilation times, ICU stays and postoperative hospital stay were defined as early secondary endpoints. Respiratory failure was defined as severe hypoxemia and difficult weaning from the ventilator or tracheal re-intubation. Delayed awaking was defined as regaining consciousness after 48 hours, excluding re-sedation due to tracheal re-intubation or unplanned reoperation.

Follow-up data were obtained from outpatient visits combining with telephoning patients. The first imaging review was performed before hospital discharge. It was recommended that patients undergo aortic computed tomography angiography (CTA) examinations at 3 months after the surgery and annually thereafter. The long-term endpoints included late death, aortic related reintervention and adverse aortic events [aortic rupture, endoleaks, retrograde type A dissection (RTAD), stent migration and fracture and etc.].

The study was conducted in accordance with the Declaration of Helsinki (as revised in 2013). The study was approved by institutional ethics committee of Fuwai Hospital (No. 2018-1069) and informed consent was waived.

\section{Statistical analysis}

Statistical analyses were performed using SPSS, version 21.0 (SPSS, Inc., Chicago, IL, USA). Descriptive statistics for categorical variables are reported as the frequency and percentage, whereas continuous variables are reported as the mean \pm standard deviation $(\mathrm{SD})$ or median with interquartile range (IQR) depending on a normal distribution. For evaluation of the early- and long-term outcomes, patients were stratified into two subgroups by 65 years. Coincidentally, there were 18 patients in both subgroups. Propensity score matching for the two subgroups was not used because demographic characteristics, such as spectrum of pathology and comorbidities are certainly different for 
Table 1 Demographic characteristic

\begin{tabular}{|c|c|}
\hline Variables & All patients $(n=36)$ \\
\hline Age (years) & $63.6 \pm 9.3$ \\
\hline Male sex, n (\%) & $33(91.7)$ \\
\hline BMI $\left(\mathrm{kg} / \mathrm{m}^{2}\right)$ & $24.56 \pm 4.20$ \\
\hline \multicolumn{2}{|l|}{ Diagnosis, n (\%) } \\
\hline Aneurysm & $12(33.3)$ \\
\hline TBAD & $9(25.0)$ \\
\hline PAU & $8(22.2)$ \\
\hline Pseudoaneurysm & $3(8.3)$ \\
\hline Complications after TEVAR & $3(8.3)$ \\
\hline $\mathrm{IMH}$ & $1(2.8)$ \\
\hline \multicolumn{2}{|l|}{ Medical history, n (\%) } \\
\hline Hypertension & $30(83.3)$ \\
\hline CAD & $12(33.3)$ \\
\hline Diabetes mellitus & $5(13.9)$ \\
\hline Peripheral arterial disease & 5 (13.9) \\
\hline Old CVA & $4(11.1)$ \\
\hline CKD & $2(5.6)$ \\
\hline COPD & $1(2.8)$ \\
\hline Smoking & $22(61.1)$ \\
\hline Previous aortic surgery, n (\%) & $4(11.1)$ \\
\hline TEVAR & $3(8.3)$ \\
\hline AAA resection & $1(2.8)$ \\
\hline EuroScore system & $8.22 \pm 2.98$ \\
\hline Intermediate risk, n (\%) & $6(16.7)$ \\
\hline High risk, n (\%) & $30(83.3)$ \\
\hline
\end{tabular}

BMI, body mass index; TBAD, type B aortic dissection; PAU, penetrating aortic ulcer; TEVAR, thoracic endovascular aortic repair; IMH, intramural hematoma; CAD, coronary artery disease; CVA, cerebral vascular accident; CKD, chronic kidney disease; COPD, chronic obstructive pulmonary disease; AAA, abdominal aortic aneurysm.

elderly and young patients. Categorical variables were compared with Fisher's exact test and continuous variables were compared with Student's $t$-test or Mann-Whitney U test as appropriate. Long-term survival rates, late aortic related reintervention and adverse aortic events rates were analyzed using a Kaplan-Meier survival curve and log-rank tests.

\section{Results}

\section{Baseline characteristics}

The mean age of all 36 patients was $63.6 \pm 9.3$ years and $91.7 \%(n=33)$ of them were male. Aortic pathology of these patients is various, including $12(33.3 \%)$ aneurysm, 9 (25.0\%) type B aortic dissections (TBAD), 8 (22.2\%) penetrating aortic ulcer (PAU), $3(8.3 \%)$ aortic arch pseudoaneurysm, 3 (8.3\%) complications after thoracic endovascular aortic repair (TEVAR) for TBAD. One (2.8\%) intramural hematoma. Depending on EuroScore system, 30 $(83.3 \%)$ patients were stratified into high risk level. Other baseline characteristics were shown in the Table 1 with detail.

\section{Operative characteristics}

Operative characteristics were described in Table 2 with detail. Retrograde stents deployment was succeeded in $29(80.6 \%)$ patients. The median proximal diameter of stents was 34 (IQR: $32-35.5$ ) $\mathrm{mm}$, and mean oversize rate of proximity was $12.9 \%$. The distal landing zone ranged from T6 to T12. A total of 10 patients underwent other cardiac or vascular surgery during the operation. On-pump operation were performed in $5(13.9 \%)$ patients, all of which were for concomitant operations.

\section{Early clinical outcomes of single-stage type I bybrid total aortic arch repair}

All patients acquired technical success and survived from the surgery. Thirty-two (88.9\%) patients were discharged from hospital without early complications. As is shown in the Table 3, severe early complications occurred in four patients, which constituted an $11.1 \%$ (4/36) of composite adverse events rate. In-hospital mortality was $8.3 \%(3 / 36)$ and 30 -day mortality was $5.6 \%(2 / 36)$. Detail of those four patients was described as follows. The first case died of stroke at 50 days after surgery. The second case died of MODS caused by mediastinal and pulmonary infection at 28 days after surgery. During his hospital stay, CRRT was used for ARF and reoperation was performed for mediastinal debridement. RTAD was occurred at the third case at 5 days after operation and this patient died shortly after the emergency reintervention. The fourth case survived from respiratory failure and transferred to nursing home at 21 days after the operation. CTA examination before discharge was performed at 32 patients and CT plain 
Table 2 Operative characteristics

\begin{tabular}{|c|c|}
\hline Variables & All patients $(n=36)$ \\
\hline Operation time (hours) & $4.5(4.0-5.5)$ \\
\hline \multicolumn{2}{|c|}{ Patterns of supra-arch debranching, $\mathrm{n}(\%)$} \\
\hline Patterns 1 & $27(75.0)$ \\
\hline Patterns 2 & $4(11.1)$ \\
\hline Patterns 3 & $5(13.9)$ \\
\hline \multicolumn{2}{|l|}{ Delivery approach, n (\%) } \\
\hline Retrograde & $29(80.6)$ \\
\hline Antegrade & $7(19.4)$ \\
\hline \multicolumn{2}{|l|}{ Number of stents, $\mathrm{n}(\%)$} \\
\hline 1 & $20(55.6)$ \\
\hline 2 & $15(41.7)$ \\
\hline 4 & $1(2.8)$ \\
\hline \multicolumn{2}{|l|}{ Oversize of proximity, n (\%) } \\
\hline$<10 \%$ & $3(8.3)$ \\
\hline $10-15 \%$ & $26(72.2)$ \\
\hline $15-20 \%$ & $7(19.4)$ \\
\hline \multicolumn{2}{|l|}{ Distal landing zone, n (\%) } \\
\hline$T^{\mathrm{a}} 6^{\mathrm{b}}$ & $3(8.3)$ \\
\hline $\mathrm{T} 7$ & $13(36.1)$ \\
\hline T8 & 5 (13.9) \\
\hline T9 & $10(27.8)$ \\
\hline T10 and more distal & $5(13.9)$ \\
\hline On pump surgery, $\mathrm{n}(\%)$ & $5(13.9)$ \\
\hline Concomitant procedures, n (\%) & $10(27.8)$ \\
\hline CABG & $9(25.0)$ \\
\hline Valve surgery & $2(5.5)$ \\
\hline Peripheral artery surgery & $3(8.3)$ \\
\hline Intraoperative transfusion, $\mathrm{n}(\%)$ & $14(38.9)$ \\
\hline
\end{tabular}

a, abbreviation of "thoracic vertebrae"; ${ }^{\text {b }}$, the number means the segment of thoracic vertebra. CABG, coronary artery bypass graft.

examination was performed at the other four patients with severe operative complications. Besides RTAD was diagnosed in $1(1 / 36,2.8 \%)$ patients by clinical feature and operation exploration, other stents and bypass complications were not observed during hospital stay.
Significant difference was not observed on early clinical outcomes between two subgroups.

\section{Long-term clinical outcomes of single-stage type I bybrid total aortic arch repair}

In the follow-up study, 3 (8.3\%) patients died during hospital stay. One (3.0\%) patient was loss of follow-up at 10 months. The average follow-up time was $48.0 \pm 35.3$ months (max: 124 months). During follow-up periods, late death occurred at $6(18.2 \%, 6 / 33)$ patients, $3(9.1 \%, 3 / 33)$ of whom were aortic related. Detail of six dead patients were shown in Table 4 with detail. Overall survival rate was $83.3 \%$ and $51.9 \%$ at 5 and 10 years respectively. The 5 -year survival rate were $88.9 \%$ and $77.8 \%(\mathrm{P}=0.41)$ in the elderly and young groups, respectively. Kaplan-Meier long-term survival curves are shown in Figure $2 A, 2 B$. One patient underwent late aortic related reintervention (total arch replacement with frozen elephant trunk) for RTAD at 7 years after the first operation and died after reintervention. Kaplan-Meier freedom from aortic related reintervention curves in Figure 2C,2D and significant difference was not observed between two subgroups $(\mathrm{P}=0.92)$. Late adverse aortic events were observed at seven $(21.2 \%, 7 / 33)$ patients. Sudden aortic rupture was noticed at two patients, RTAD was noticed at one patient, new emerging type A aortic dissection (TAAD) was noticed at one patient, endoleaks (all were type Ia) were noticed at three patients, but hemodynamic significance was not observed. Stents fracture, migration, infection or bypass graft occlusion was not observed during follow-up periods. Detail of late adverse aortic events were described at Table 5. Overall freedom from adverse aortic events rate was $79.2 \%$ and $47.5 \%$ at 5 and 10 years respectively. The 5 -year freedom from adverse aortic events rate were $93.3 \%$ and $67.0 \%$ $(\mathrm{P}=0.06)$ in the elderly and young subgroups, respectively. Kaplan-Meier freedom from adverse aortic events curves are shown in Figure 2E,2F. CTA images of these patients with late adverse aortic events were presented at Figure 3.

\section{Discussion}

Aortic surgery has acquired great progress since the first aortic operation in 1950s (10), but is still a challenging problem in the cardiovascular surgery. Total arch replacement with frozen elephant trunk, reported by Sun, has become a routine procedure for extensive aortic arch disease in China (11). However, HCA greatly impacts on 
Table 3 Early clinical outcomes of single-stage type I hybrid total aortic arch repair

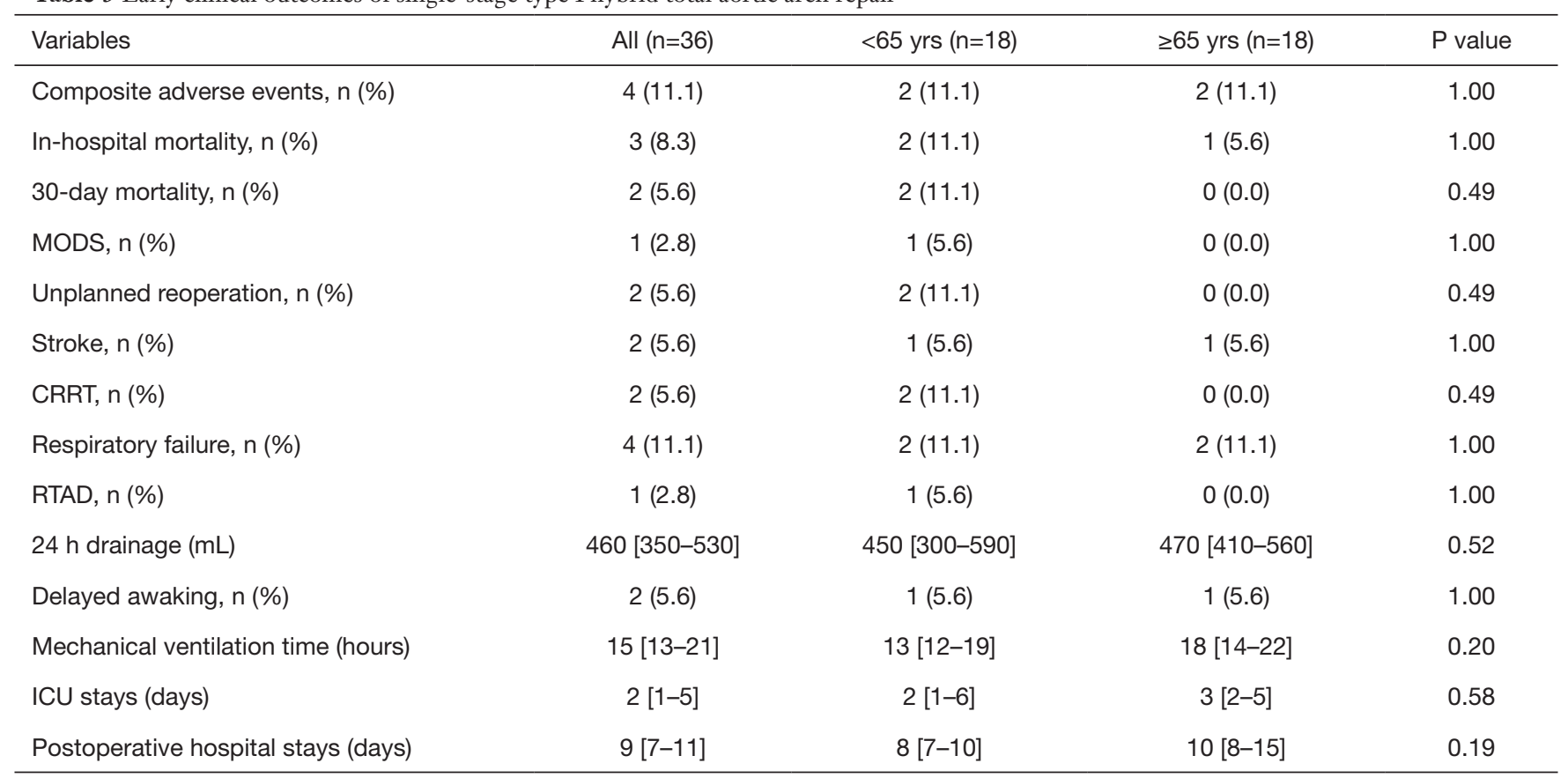

MODS, multiple organ dysfunction syndrome; CRRT, continuous renal replacement therapy; RTAD, retrograde type A dissection; ICU, intensive care unit.

Table 4 Detail of six late dead patients

\begin{tabular}{lcccc}
\hline Patients & Sex & Age $^{\text {a }}$ (years) & Survival periods & Cause of death \\
\hline Case 1 & Male & 56 & 2 months & Sudden aortic rupture \\
Case 2 & Male & 62 & 8 years & Sudden aortic rupture \\
Case 3 & Male & 66 & 7 years & Reintervention for RTAD \\
Case 4 & Male & 64 & 5.5 years & Acute myocardial infarction \\
Case 5 & Male & 79 & 3 months & Pneumonia and heart failure \\
Case 6 & Male & 59 & 5 months & Cancer \\
\hline
\end{tabular}

${ }^{\text {a }}$, age at the time of the surgery. RTAD, retrograde type A dissection.

perioperative safety, as a result, which is unsuitable for elderly or high-risk patients. CPB and HCA were both avoided in the type I hybrid total aortic arch repair, making it mini-invasive.

Although type I hybrid total aortic arch repair has many advantages, strict surgical indications hinder its clinical practice. Aortic dissection is more prevailing in Chinese aortic pathology spectrum, whereas TAAD is a contraindication for this surgery. Except those patients with ascending aortic dissection, many elderly patients diagnosed with aneurysm often have ascending aortic expansion or plaque, which also excluded many candidates for this surgery. Therefore, although this operation is safe, the indications are too strict and the cost is relatively expensive, which explains why there were so less cases underwent this surgery in our hospital during the past 10 more years.

Ten more years ago, Rizvi reported that very lowquality evidence suggests that LSCA coverage increases the risk of neurological complications (12). Cooper reported that preemptive revascularization of LSCA offered no protection against cerebral vessel accident (13). Guidelines from Society for Vascular Surgery (SVS) also recommended 
A

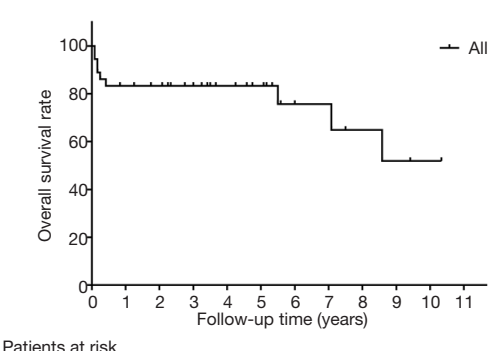

Patients at risk

$\begin{array}{lllllllllll}36 & 30 & 27 & 22 & 18 & 15 & 8 & 8 & 6 & 5 & 2\end{array}$

\section{B}

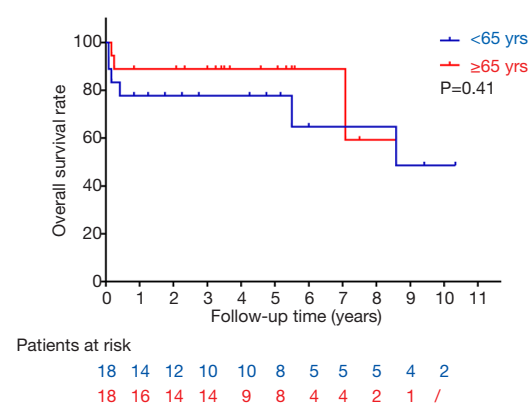

C

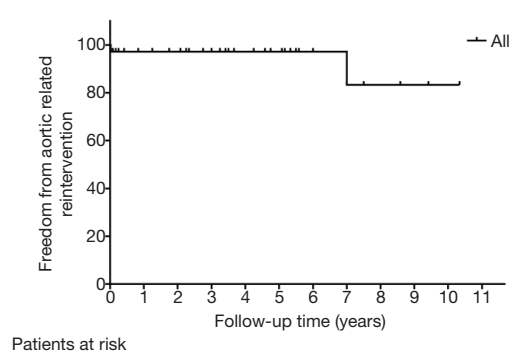

$\begin{array}{lllllllllll}36 & 30 & 27 & 22 & 18 & 15 & 8 & 7 & 6 & 5 & 2\end{array}$

D

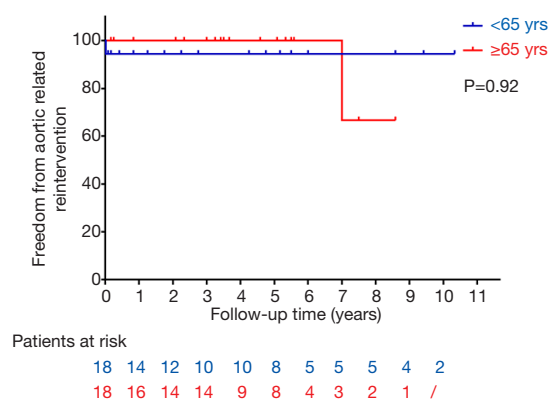

$\mathrm{E}$

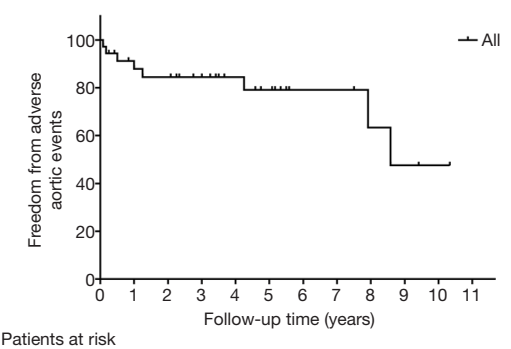

Patients at risk

$\mathrm{F}$

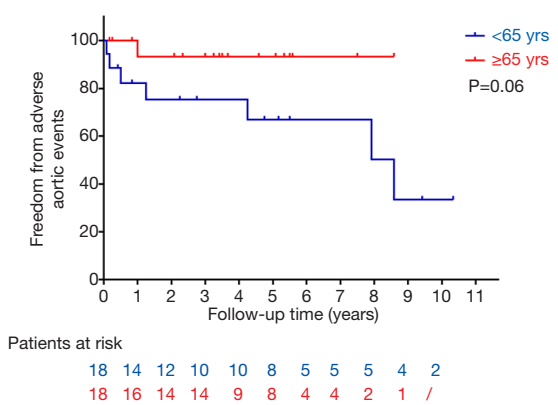

Figure 2 Kaplan-Meier survival analysis and log-rank tests for long-term outcomes of single-stage type I hybrid total aortic arch repair. (A) Kaplan-Meier survival curves for all patients. (B) Kaplan-Meier survival curves for elderly (red line) and young (blue line) patients. (C) Rate of freedom from aortic related reintervention for all patients. (D) Rate of freedom from aortic related reintervention for elderly (red line) and young (blue line) patients. (E) Rate of freedom from adverse aortic events for all patients. (F) Rate of freedom from adverse aortic events for elderly (red line) and young (blue line) patients.

Table 5 Detail of seven patients with late adverse aortic events

\begin{tabular}{lccccc}
\hline Patients & Sex & Age $^{\text {a }}$ (years) & Complications & Survival periods & Management and prognosis \\
\hline Case 1 & Male & 56 & Aortic rupture & 2 months & Sudden death without emergency treatment \\
Case 2 & Male & 62 & Aortic rupture & 8 years & Sudden death without emergency treatment \\
Case 3 & Female & 54 & TAAD & 8 years & Periodical CTA examination and survival \\
Case 4 & Male & 66 & RTAD & 1 year & $\begin{array}{c}\text { Enlargement of false lumen was observed during follow-up and } \\
\text { reintervention was performed at } 7 \text { years, and died after reintervention }\end{array}$ \\
Case 5 & Male & 47 & la endoleaks & 15 months & Periodical CTA examination and survival \\
Case 6 & Male & 55 & la endoleaks & 4 years & Periodical CTA examination and survival \\
Case 7 & Male & 58 & la endoleaks & 6 months & Periodical CTA examination and survival
\end{tabular}

a , age at the time of the surgery. TAAD, type A aortic dissection; RTAD, retrograde type A dissection; CTA, computerized tomography angiography. 

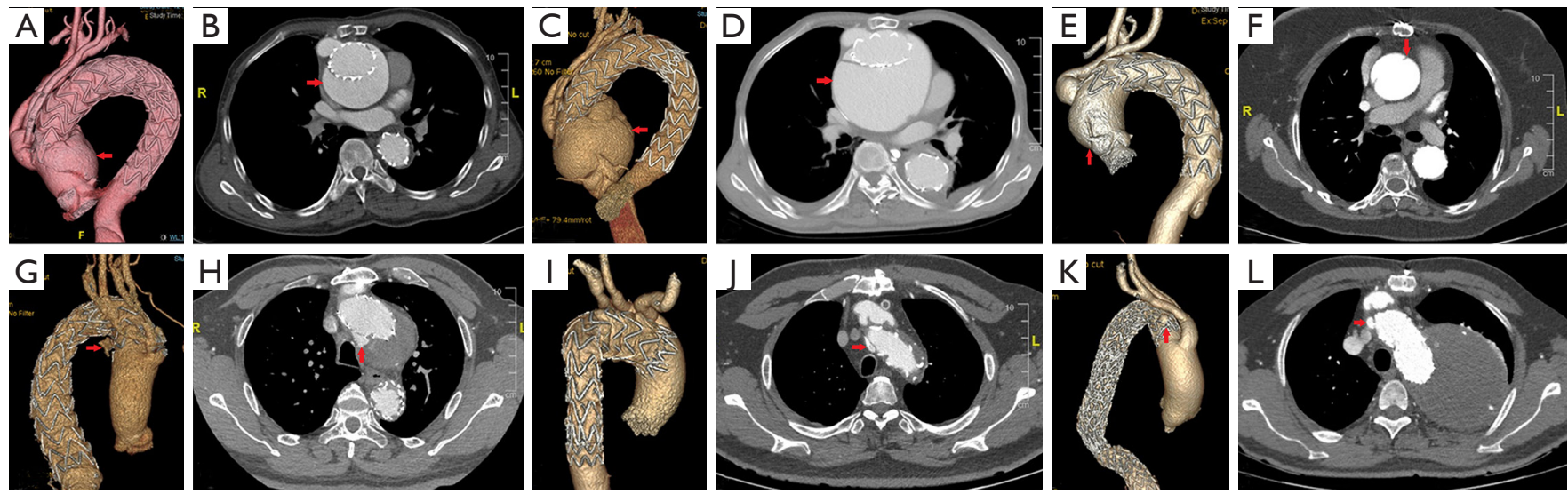

Figure 3 CTA images of late adverse aortic events. (A,B) CTA images noticed RTAD in one patient at 1 year after operation. (C,D) CTA images noticed enlarging false lumen of RTAD in that patient at 7 years after operation, also before reintervention, comparing with (A,B). (E,F) CTA images noticed new emerging TAAD in one patient at 8 years after operation. (G-L) CT images noticed type Ia endoleaks in three patients at 4 years, 6 months and 15 months after operation, respectively. CTA, computed tomography angiography; RTAD, retrograde type A dissection; TAAD, type A aortic dissection.

revascularization of LSCA should be individualized in highrisk patients (14). Therefore, LSCA was not revascularized in the early stages of developing this surgery to shorten operative time and improve operative safety. Thereafter, increasing number of studies had reported that LSCA revascularization may lower the rate of stroke (15-18). With the improvement of surgical skills and accumulation of experience, LSCA revascularization has become routine since 2013 in our center. Fortunately, neurologic complications were not observed in these five patients without LSCA revascularization.

Although organ protection of cardiovascular surgery has made great progress, CPB and HCA are no doubt risk factors for perioperative morbidity and even mortality. From STS Database, the operative mortality and frequency of the composite endpoint of operative mortality and neurologic complication of 12,521 patients underwent aortic arch repairs with HCA between 2011 to 2014 were $12 \%$ and $23 \%$, respectively (1). In China, Wang reported that 30-day mortality of 1,708 patients who underwent aortic arch surgery with HCA between 2009 and 2015 was $6.1 \%$, but the average age and comorbidity of these patients was lower (2). For type II hybrid total aortic arch repair, HCA is avoided, but CPB is still inevitable. Reported by Fuwai Hospital, early mortality of 122 patients underwent type II hybrid repair for TAAD was 9.2\% (19). Compared with early clinical outcomes of other types of aortic arch repair under CPB or HCA, single-stage type I hybrid total aortic arch repair in our center is safe at perioperative periods. Previous study reported the early mortality of type I hybrid total arch repair were $11 \%$ (6), $8.3 \%$ of early mortality for elderly or high-risk patients in our center was also desirable. Significant difference was not observed by subgroup analysis, so we speculate that type I hybrid repair is mini-invasive and does not increase the perioperative risks in the elderly or high-risk patients.

Although endovascular repair improves perioperative safety, the durability of stents concerns the surgeons. Endovascular repair has been widely used in the era of descending aorta disease, and achieved considerable clinical outcomes $(3,20)$. Because descending aorta is straight but aortic arch is curved, so the operative difficulty and durability of endovascular aortic arch repair will be impacted. Due to the short history and strict indications of type I hybrid total aortic arch repair, so there was less clinical study with large samples and long-term follow-up worldwide. Joo reported that for all types of hybrid aortic arch repair, the adverse aortic event-free rates at 6 years were $39.4 \% \pm 10.3 \%$, and zone 0 hybrid aortic arch repair has higher adverse aortic events rate (6). With average nearly 5-year follow-up period, we consider long-term clinical outcomes in our center are desirable and there are two reasons for these outcomes. Firstly, proximity of the endovascular stent was deployed to the place that just cover the innominate artery ostia rather than particularly close to the proximity of ascending aorta as reported by other 
studies $(5,6,9)$. In this manner, on the basis of effectively blocking diseases aorta and supra-arch branches, the angle of the stent can be reduced, thus reducing the pressure on the aortic wall from both ends of the stents. Secondly, strict patient selecting is also an important factor to guarantee the long-term clinical outcomes.

Between the two subgroups, late survival rate and freedom from reintervention rate are comparable, meanwhile, freedom from adverse aortic events rate is lower in the young subgroup, but significant difference was not observed. With the extension of follow-up time and the expansion of sample size, we consider that significant difference will be likely observed. Elderly patients with short life expectancy may not suffer from adverse aortic events before they died of other disease. For younger patients, movements are more common, which may influence the pressure between the aorta and the stents. In this background, stent complications will be more prevailing, but no previous study reported. Many elderly patients are unable to come to Fuwai Hospital for CTA examination due to their advanced age or comorbidities, meanwhile, the quality of primary health care in China was not decent (21). Therefore, the diagnostic rate of mild endoleaks may be biased and influence the incidence of adverse aortic events.

\section{Limitation}

Retrospective study design at a single center and small sample size may cause to some bias in the results. In the future, prospective research, expanding the sample size and continuously prolonging follow-up time will be necessary.

\section{Conclusions}

With the past 10 more years of experience, single-stage type I hybrid total aortic arch repair for extensive aortic arch disease has achieved desirable outcomes in our center. Because of avoiding CPB and even HCA, perioperative safety is improving, which give those elderly or highrisk patients suffering from extensive aortic arch disease an opportunity to undergo surgical treatment. For young patients with longer life expectancy diagnosed with extensive aortic arch disease, long-term outcomes were also acceptable, so we consider this surgery is an option, but not primary option for younger patients. In conclusion, with limited experience, single-stage type I hybrid total aortic arch repair is a practical mini-invasive treatment for extensive aortic arch disease, but surgical indications should be strictly controlled, which will optimize the early- and long-term outcomes of this surgery.

\section{Acknowledgments}

We appreciate Liang Xian (Bengbu Medical College, Anhui, China) for drawing those pictures showing operative technique.

Funding: This work was supported by Beijing Municipal Science and Technology Commission (Z181100001718197).

\section{Footnote}

Reporting Checklist: The authors have completed the TREND reporting checklist. Available at http://dx.doi. org/10.21037/jtd-20-3479

Data Sharing Statement: Available at http://dx.doi. org/10.21037/jtd-20-3479

Conflicts of Interest: All authors have completed the ICMJE uniform disclosure form (available at http://dx.doi. org/10.21037/jtd-20-3479). All authors report grants from Beijing Municipal Science and Technology Commission, during the conduct of the study.

Ethical Statement: The authors are accountable for all aspects of the work in ensuring that questions related to the accuracy or integrity of any part of the work are appropriately investigated and resolved. The study was conducted in accordance with the Declaration of Helsinki (as revised in 2013). The study was approved by institutional ethics committee of Fuwai Hospital (No. 2018-1069) and informed consent was waived.

Open Access Statement: This is an Open Access article distributed in accordance with the Creative Commons Attribution-NonCommercial-NoDerivs 4.0 International License (CC BY-NC-ND 4.0), which permits the noncommercial replication and distribution of the article with the strict proviso that no changes or edits are made and the original work is properly cited (including links to both the formal publication through the relevant DOI and the license). See: https://creativecommons.org/licenses/by-nc-nd/4.0/.

\section{References}

1. Englum BR, He X, Gulack BC, et al. Hypothermia and 
cerebral protection strategies in aortic arch surgery: a comparative effectiveness analysis from the STS Adult Cardiac Surgery Database. Eur J Cardiothorac Surg 2017;52:492-8.

2. Wang X, Yang F, Zhu J, et al. Aortic arch surgery with hypothermic circulatory arrest and unilateral antegrade cerebral perfusion: perioperative outcomes. J Thorac Cardiovasc Surg 2020;159:374-87.e4.

3. Chiu P, Goldstone AB, Schaffer JM, et al. Endovascular versus open repair of intact descending thoracic aortic aneurysms. J Am Coll Cardiol 2019;73:643-51.

4. Xydas S, Mihos CG, Williams RF, et al. Hybrid repair of aortic arch aneurysms: a comprehensive review. J Thorac Dis 2017;9:S629-34.

5. Milewski RK, Szeto WY, Pochettino A, et al. Have hybrid procedures replaced open aortic arch reconstruction in high-risk patients? A comparative study of elective open arch debranching with endovascular stent graft placement and conventional elective open total and distal aortic arch reconstruction. J Thorac Cardiovasc Surg 2010;140:590-7.

6. Joo HC, Youn YN, Kwon JH, et al. Late complications after hybrid aortic arch repair. J Vasc Surg 2019;70:102330.e1.

7. Vallejo N, Rodriguez-Lopez JA, Heidari P, et al. Hybrid repair of thoracic aortic lesions for zone 0 and 1 in highrisk patients. J Vasc Surg 2012;55:318-25.

8. Canaud L, Hireche K, Berthet JP, et al. Endovascular repair of aortic arch lesions in high-risk patients or after previous aortic surgery: midterm results. J Thorac Cardiovasc Surg 2010;140:52-8.

9. Weigang E, Parker J, Czerny M, et al. Endovascular aortic arch repair after aortic arch de-branching. Ann Thorac Surg 2009;87:603-7.

10. De Bakey ME, Crawford ES, Cooley DA, et al. Successful resection of fusiform aneurysm of aortic arch with replacement by homograft. Surg Gynecol Obstet 1957;105:657-64.

11. Liu ZG, Sun LZ, Chang Q, et al. Should the "elephant trunk" be skeletonized? Total arch replacement combined with stented elephant trunk implantation for Stanford

Cite this article as: Zhang B, Wei Y, Liu Y, Lin H, Liang S, Dun Y, Yu C, Qian X, Guo H, Sun X. Safety and durability of single-stage type I hybrid total aortic arch repair for extensive aortic arch disease: early- and long-term clinical outcomes from a single center and our 10-year of experience. $\mathrm{J}$ Thorac Dis 2021;13(11):6230-6239. doi: 10.21037/jtd-20-3479 type A aortic dissection. J Thorac Cardiovasc Surg 2006;131:107-13.

12. Rizvi AZ, Murad MH, Fairman RM, et al. The effect of left subclavian artery coverage on morbidity and mortality in patients undergoing endovascular thoracic aortic interventions: a systematic review and meta-analysis. J Vasc Surg 2009;50:1159-69.

13. Cooper DG, Walsh SR, Sadat U, et al. Neurological complications after left subclavian artery coverage during thoracic endovascular aortic repair: a systematic review and meta-analysis. J Vasc Surg 2009;49:1594-601.

14. Matsumura JS, Lee WA, Mitchell RS, et al. The Society for Vascular Surgery Practice Guidelines: management of the left subclavian artery with thoracic endovascular aortic repair. J Vasc Surg 2009;50:1155-8.

15. Patterson BO, Holt PJ, Nienaber C, et al. Management of the left subclavian artery and neurologic complications after thoracic endovascular aortic repair. J Vasc Surg 2014;60:1491-7.e1.

16. Waterford SD, Chou D, Bombien R, et al. Left subclavian arterial coverage and stroke during thoracic aortic endografting: a systematic review. Ann Thorac Surg 2016;101:381-9.

17. Bradshaw RJ, Ahanchi SS, Powell O, et al. Left subclavian artery revascularization in zone 2 thoracic endovascular aortic repair is associated with lower stroke risk across all aortic diseases. J Vasc Surg 2017;65:1270-9.

18. Huang Q, Chen XM, Yang H, et al. Effect of left subclavian artery revascularisation in thoracic endovascular aortic repair: a systematic review and meta-analysis. Eur J Vasc Endovasc Surg 2018;56:644-51.

19. Zhang L, Yu C, Yang X, et al. Hybrid and frozen elephant trunk for total arch replacement in DeBakey type I dissection. J Thorac Cardiovasc Surg 2019;158:1285-92.

20. Lederle FA, Kyriakides TC, Stroupe KT, et al. Open versus endovascular repair of abdominal aortic aneurysm. N Engl J Med 2019;380:2126-35.

21. Li X, Lu J, Hu S, et al. The primary health-care system in China. Lancet 2017;390:2584-94. 